Canadian Journal of Family and Youth, 13(3), 2021, pp. 53-63

ISSN 1718-9748@ University of Alberta

http://ejournals,library,ualberta.ca/index/php/cjfy

\title{
The Inevitability of Workplace Romance
}

\author{
Dallas Bilyk ${ }^{1}$
}

\begin{abstract}
The existence of workplace relationships poses many consequences for professional organizations. There are many factors such as motive, age, gender, and workplace culture that impact how these complex relationships are perceived by other employees of the organization. These perceptions can have a major influence on employee engagement, workplace productivity, and general cohesiveness of the organization that can be essential to its success. A proper response from management in terms of policy development is crucial in dealing with this form of intimate relationship. The existence of relationships in the workplace is inevitable as they have become very popular locations to discover a significant other.
\end{abstract}

\footnotetext{
${ }^{1}$ MacEwan University
} 


\section{Introduction}

Romantic relationships in the workplace have been a common occurrence for many years, and initially began following an increase of women in the workforce as a result of men leaving the country to fight in the World War (Adler \& Quist, 2014, p. 329). The frequency of interaction between men and women have led to intimate workplace relations that can best be defined by Pierce et al. (1996) as a relationship, “...between two members of the same organization that entails mutual sexual attraction" (p. 6). These are important foundational concepts to understand how romance in the workplace has been both institutionalized within organizations and normalized in society. There are many factors that contribute to either the positive or negative aspects of engaging in workplace relationships (WRs) including co-worker reactions, workplace productivity, and employee engagement. The individuals involved in a WR often times are disproportionately affected by the consequences of the relationship as a result of their gender or position within the organization. The presence of these intimate relationships within the workplace exist in a variety of forms depending on how exclusive the couple is, the publicity of the relationship or the policies that exist in regard to intimate relationships at work. Although the majority of research on this topic concludes that the consequences outweigh the benefits it is important to understand that this form of relationship is not going to disappear anytime soon as the workplace has become an extremely common place to find romantic partners. In this paper I will discuss the implications that arise from this form of intimate relationship in the workplace and how multiple aspects of the organization and its employees are impacted by two co-workers entering a romantic relationship.

\section{Literature Review}

A major trend that I discovered during my research was that of the individual status of each co-worker involved in the WR, and whether or not the relationship was considered hierarchal or lateral (Pierce \& Aguinis, 1997, p. 198). Every source that I cited included a thorough discussion regarding the power differential that can exist within a WR between a superior and a subordinate and the negative reactions that commonly occur as a result. Within the two types of WRs another aspect that was commonly discovered to impact co-worker perceptions was that of motive, in which if an individual chose to engage in a relationship for love, ego, or job opportunity they will be perceived differently by co-workers (Shuck, Owen, Manthos, Quirk, \& Rhoades, 2016; Wilson, 2015). If one of the individuals involved in the WR is a female they will often times experience more negativity for their participation in the relationship compared to their male counterpart (Cowan \& Horan, 2014; Pierce, Byrne, \& Aguinis, 1996; Alder \& Quist, 2014; Salvaggio, Hopper, \& Packell, 2010). The disproportionate gender evaluations of WRs in regard to women further perpetuates the inequalities that exist for them in the workplace that have been structured into our society. The consequences that exist due to the presence of WRs in an organization do lead many researchers to conclude that there should be policies that are created by management to deal with relationships if there is a break-up or the uncomfortable environment the relationships could develop in regard to other co-workers feelings (Alder \& Quist, 2014; Balaban, 2019; Cowan \& 
Horan, 2014; Wilson, 2015). It is suggested that the relationships should be dealt with individually as each partnership will be vastly different in terms of status, intention, co-worker awareness and commitment level.

Despite the multitude of consequences and negative perceptions that result from employee relationships there are positives that also exist alongside them. There are personal benefits that exist for the couple, co-workers who support the relationship, and a suggested increase in workplace group cohesiveness (Alder \& Quist, 2014; Chroy \& Hoke, 2019; Cowan \& Horan, 2014; Salvaggio, Hopper, \& Packell, 2010). The presence of positive reactions of coworkers will be dependent on the age of the employees as well as the workplace culture that already exists. This would most likely mean that if there was already a pre-existing negative work environment the reactions of employees to a WR would be equally as negative and discouraging to the continuance of the relationship. There is much contemporary research that still needs to be done on this topic, as many of the sources that are available are dated or utilize information and research that was conducted years ago. Much of society in regard to work has changed as it is no longer a new concept of women being in the work force. With much of the older generation that started to see the shift in labour after the world war retire, a new generation of more tolerant and flexible millennials are taking over the workplace organizations (Chroy \& Hoke, 2019). It is essential to also do research surrounding the existence of homosexual relationships at work and how co-worker reactions may differ from that of heterosexual relations. Although there are comments in multiple of my references on management policy development in regard to WRs it is quite clear that no concrete answer exists about what organizations should do in response to their employees engaging in relationships. This is a complex topic that affects many people who work, and it requires more modern academic research.

\section{Hierarchal vs. Lateral Relationships}

As best defined by Fiona Wilson (2015), hierarchal relationships are when one of the two employees are at a higher organizational level than the other, while lateral relationships are between employees of equal status (p. 2). There are many complications that occur within the WRs as a result of the power differentials in which co-workers perceive that the subordinate in the relationship gains an unfair advantage for being involved in the relationship and is favoured by the individual in power in regard to workplace tasks. The subordinate has access to the superior power that other co-workers do not, which leads to the assumptions that they will use this to their advantage in terms of organizational opportunity (Malachowski, Chroy, \& Claus, 2012, p. 373). Lateral relationships tend to cause much less disruption in the workplace and can even cause a sort of sexual electricity in the work environment that excites co-workers, and as a result boosts their morale (Pierce, Byrne, \& Aguinis, 1996, p. 20). It is the perceived disadvantage of other employees, in which they anticipate at some point they will not be treated fairly in the organization as they are not in a hierarchal relationship that make them much less acceptable and positively considered (Alder \& Quist, 2014, p. 334). 


\section{Motives}

A major part of research on the topic of WRs is that of motive for involvement. There are three motives that are often identified throughout multiple sources including love, ego, and job. In her research, Wilson (2015), clearly establishes what each motive entails in which someone with a love motive is seeking a long-term and committed companionship, an individual with a motive of ego is seeking the thrill, excitement, sexual experience or satisfaction that a WR may bring, and finally someone with a job motive is seeking advancement, security, power, and financial reward for their participation in a WR (Malachowski, Chroy, \& Claus, 2012, p. 360; Wilson, 2015, p. 5). The motive of the relationship is typically what dictates how co-workers will respond to its existence. If the motive for entering a WR is selfish this fact can affect many parts of the organization including employee engagement, in which the focus of the employee shifts from their job to the maintenance of their relationship and what they desire to gain from being involved (Shuck, Owen, Manthos, Quirk, \& Rhoades, 2016, p. 384). This leads to co-workers feeling threatened, and less trusting of the employees involved in the WR which often leads to a higher probability of vicious gossip which can aid in creating a toxic workplace environment. This is mostly the case for subordinates dating superiors in that it is a common belief that they are much less likely to be in the relationship for love, but rather involved in order to attain a goal of some sort (Malachowski, Chroy, \& Claus, 2012, p. 373-374).

\section{Workplace Culture}

The types of behaviours that are tolerated in a specific organization will correlate to the prevalence of WRs. This includes a variety of factors such as long work hours, flirting, joking, and provocative dressing (Wilson, 2015, p. 5). Workplaces that are considered slow, traditional, and conservative have lower occurrences of WR, while on the other hand organizations that are dynamic, busy, and liberal encourage sexual behaviours (Pierce, Byrne, \& Aguinis, 1996, p. 16). Wilson (2015) uses the example of a bank environment opposed to a restaurant or advertising when illustrating the differences in workplace culture and further discusses how this type of behaviour in the workplace has the potential to cause substantial harm to many employees. This is because every individual experience's sexual advancements in a different manner whether it is wanted or unwanted (p. 5). The frequency of contact between individuals within workplace organizations causes the WR to be as, "...inevitable as earthquakes in California” in which people begin to grow fond of one another as a result of close working quarters (Balaban, 2019, p.127). Some individuals may even cross the line in the workplace and take sexual advancements too far in which it begins to be identified as sexual harassment. In organizations that do not set out to create a policy in regard to WRs may see themselves faced with sexual harassment claims which often involve superior-subordinate relationships, as the power differential has the potential to create hostility and resentment between the couple (Pierce \& Aguinis, 1997, p. 199). 


\section{Gender}

Women who choose to be a part of a WRs will in most instances have a more negative experience in terms of reaction from co-workers and face more job insecurity if the relationship fails. In hierarchal romances specifically in which a male superior and female subordinate are involved in a relationship the female will more often be seen as disposable and more at risk of transfer and even termination, which are common tactics to dealing with break ups of a WRs (Pierce, Byrne, \& Aguinis, 1996, p. 24-25). It has been suggested that women bring sexuality into the workplace in terms of their communication and behaviours in comparison to men, and therefore women subordinates in WRs are perceived as less trustworthy and less caring than male superiors (Malachowski, Chroy, \& Claus, 2012, p. 364). Wilson (2015) discusses her findings in regard to the idea that women are often perceived as "...sleeping [their] way to the top..." in which they lie and use sex to attain what they need to in the workplace (p. 6). Her discussion on a woman's motive for being in a WR found that they are believed to be involved only to move up in the organizational hierarchy and are therefore referred to as 'sluts' or 'tarts', but there is no similar belief when it comes to the men who are part of the relationship. These findings contribute to the fact that women still face disproportional discrimination in the workplace due to the structural aspects of our western society that put a higher value on men in the workplace that allows them to have much more personal freedom to do as they please in most domains that they exist in. In terms of perception of WRs researchers have discovered that women often consider intimate relationships in the workplace less positively compared to men. Salvaggio et al. (2010) states,

"...female sexuality is controlled and supressed in Western culture, whereas male sexuality is not as tightly monitored", this results in women feeling less sex positive and more likely to oppose sexual behaviours at work (p. 608).

\section{Age}

Chory and Hoke (2019) discuss multiple aspects of age in the modern workplace that exist in regard of millennials both entering and soon becoming the majority in the workplace. They discovered that millennials are less likely to oppose WRs and more likely to be romantically involved with a superior or a co-worker (p. 595). The researchers also point out that millennials commonly engage in more casual WRs in which there is little expectation in terms of commitment and can even be considered hook-ups (p. 576). They also describe how millennials expect flexibility in workplace relations, and do not prefer traditional formations of an organization in which the lines between private and personal become blended. This can cause serious complications in terms of interactions on social media where there is a risk of not knowing whether or not inappropriate communication is a workplace issue or a problem that requires action outside of work (p. 577-578). The opinions regarding the existence of relationships in the workplace is changing with the new generation of workforce and requires constant updated research as social norms change in our society. 


\section{Consequences}

Much of the research on this topic focuses on the negative consequences that exist when two employees of the same organization become involved. It has been concluded that no matter which way you look at the presence of WRs there is some level of harm that will occur throughout the entirety of the organization. The proximity and frequency of employee engagement make this inevitable. The sexual component of any type of relationship typically requires privacy, but when an employee chooses to blend both their private and personal life others around them become to feel entitled to personal information. This creates uncomfortable feelings amongst co-workers, especially when there is no formal policy that exists within the organization, so there is no standard of conduct.

\section{Co-worker Reactions}

The most influential element to the way in which WRs are perceived is other employees within the organization. An initial determining factor of whether or not the relationship will be considered negatively or positively by co-workers is the manner in which they discover the existence of the couple whether it be through personal exposure or impersonal revelations (Cowan \& Horan, 2014. p. 243). Their reactions will also be dependent on the people involved in the relationship and their status in relation to one another, their personal views on WRs, as well as the culture that the organization has established in regard to what is permissible. For instance, if there is a personal connection between the individual involved in the relationship and a co-worker in which they consider each other friends and have established trust, the WR will often be perceived positively. Trust between friends in the workplace allows for the individual in the relationship to have others to confide in and have protection from potential gossip and adverse interactions with other co-workers who may not be too fond of the WR (p. 244). On the other hand, if the WR is never explicitly presented to co-workers and is indirectly discovered much like actions that Cowan \& Horan (2014) note, "sitting close, talking often, physical closeness, and eye contact all serve as nonverbal indicators that coworkers could be more than friends" and often result in cruel gossip (p. 245). Reactions such as disbelief, surprise, and shock are also often observed when the existence of a WR is not presented formally to other co-workers and will more likely result in a negative opinion of the situation.

The status difference that exists in hierarchal relationships creates the most negative reactions amongst co-workers that impacts many aspects of the organization. If the subordinatesuperior relationships exist between a female and male respectively, issues such as favoritism and disproportionate opportunity are commonly cited by co-workers as their main concern for the existence of WRs. Employees dating superiors are seen as threatening by other co-workers because of their close connection with someone of authority, and access to potential personal benefits in the workplace which is referred to as anticipatory injustice (Alder \& Quist, 2014, p. 331). The perception of fairness will impact attitudes and behaviours of employees and as a result will affect a multitude of factors related to job performance. One reason that co-workers will interpret the WR as negative for the organization is that they look for any faults of the couple and attribute 
those faults to consequences of the relationship, when they may have no relation one another. The judgments that are formed are often not logical and exist due to preconceived notions of what a WR will entail within an organization (Alder \& Quist, 2014, p. 336).

\section{Job Productivity and Engagement}

The most damaging aspect of negative perceptions of co-workers is gossiping. The disapproval of WRs can result in hostility in which false information can potentially be spread around the workplace in regard to details of the relationship and impact both job productivity and engagement. Malachowski et al. (2012) details that co-workers may manipulate information concerning WRs in order to protect themselves due to the distrust they experience in regard to the relationship, so any information that doesn't come from the couple should be considered unreliable (p. 375). In terms of productivity Pierce et al. (1996) discuss instances of, "...excessive employee chatting, long lunches, and lengthy discussions behind closed doors" between the WR couple that have a negative effect on others within the organization (p. 19). The presence of a hierarchal relationship creates not only less productivity of the couple but also other employees who waste a substantial amount of time gossiping about the superior-subordinate relationship in regard to their potential unfair treatment. Those employees who were seeking to increase their status within the organization through participating in a WR displayed lower levels of employee engagement which is directly related to job performance (Shuck, Owen, Manthos, Quirk, \& Rhoades, 2016, p. 383). This can be a result of two aspects discussed by Shuck et al. (2016) which include that the employee may have been disengaged to begin with because they have no desire to work hard or their disengagement may be because of negative consequences they experience from other coworkers perceptions (p. 388).

Malachowksi et al. (2012) discusses the damages that can occur due to WRs which include, "...lower job satisfaction and commitment, and higher absenteeism and turnover among employees" (p. 375). It is suggested that couples in WRs should attempt to build associations with their colleagues in order to enhance their workplace environment if they choose to blend both their private and personal life together. The issues for individuals of blending both their public and private life include conflicts spilling over into the workplace which can often create awkward and uncomfortable situations for other employees in the organization due to lack of sufficient boundaries established by the couple (Chroy \& Hoke, 2019, p. 587).

\section{Sexual Harassment}

A major consequence of hierarchal relationships in the workplace is sexual harassment. As previously discussed, issues regarding workplace culture impact what is distinguished as sexual harassment depending on what behaviours have been determined acceptable. Pierce et al. (1997) thoroughly discusses the implications that exist as a result of WRs and how dissolved hierarchal relationships are more likely to lead to potential instances or accusations of sexual harassment (p. 
198). This is because a subordinate's job tasks likely rely on the cooperation of the subordinate and the resulting power differential may result in "...sexual coercion/or discriminatory managerial decision making" (p. 199). As a retaliation the subordinate could make accusations of sexual misconduct in order to get back at their superior for a variety of potentially personal reasons (Cowan \& Horan, 2014, p. 251). The manner in which the relationship ended, and the state of the relationship determines whether or not there is potentially a threat for sexual harassment allegations.

\section{Benefits}

Despite the large amounts of consequences that exist due to WRs within organizations there are aspects that exist that researchers have found to be positive. Couples may be compelled to work harder and become more involved in their workplace in a way to respond to negative opinions of colleagues. There may also be an increase in performance, in creative tasks specifically, and punctuality as a result anticipatory injustice which was previously discussed (Chroy \& Hoke, 2019, p. 579). Essentially, if a workplace is able to be categorized by a culture of care and support, they will potentially see, “...enhanced teamwork and morale, and healthier, more satisfied employees" (p. 579). If positive evaluations from employees are present in regard to romantic relationships in the workplace it has been observed that there will be an increase in group identification as well as cohesiveness because co-workers are able to accept the decision of the couple to remain together opposed to disagreeing with their choice (Alder \& Quist, 2014, p. 339). If a WR were to result in marriage this could also be seen as a benefit because as Wilson (2015) states, “...marriage could help individuals to work to their maximum potential, as their personal needs are being satisfied..." as a result of the union (p. 8).

\section{Policy and Management}

It is apparent that the presence of WRs within organizations is here to stay, they have become a common place for individuals to meet their significant others mainly as a result of frequency of contact. The negative aspects that a WR brings to the organization require policies to be developed by management so serious issues do not persist within the work environment which can affect many areas of work. There are multiple approaches that are suggested to be followed in regard to these complex and complicated relationships in which management must mainly consider how much work and personal lives are overlapping (Cowan \& Horan 2014, p. 249-250). Balaban (2019) suggests that WRs should be dealt with on a case-by-case basis as these relationships affect the workplace in varying degrees, as he suggests that if the relationship does not impact productivity it would be unfair to treat the couple the same as another who is being a destructive force (p. 133). It is also noted that issues of privacy and emotional distress can be a cause for concern in which it is not always clear when or if it is appropriate for managers to intervene in a relationship between employees. On the other hand some researchers suggest WRs should be dealt with by management soon after they learn of its existence in a manner that is considered the most 
fair by employees, and not overtly strict in order to ensure the relationship does not go 'underground' (Alder \& Quist, 2014, p. 343). The negative consequences of a failed romance should be made clear to the couple in a counselling manner that promotes integrity. In the event of a relationship between a subordinate and a superior they should be dealt with in a much tougher way as their relationship has the potential to cause much more harm within the organization. The hierarchal couple should be faced with the decision to break up or transfer out of the same department if they work directly with one another (p. 344).

A modern approach to WRs includes the understanding of a love contract in which the couple would have to read and sign a contract to indicate that the relationship is consensual, and will not cause problems for the organization in the future (Chroy \& Hoke, 2019, p. 593; Wilson, 2015 , p. 10). This constrictive form of policy is likely to be less supported by the Millennial generation that, as previously stated, values flexibility and would favour informal restrictions opposed to intrusive documents. Those values are difficult to uphold in an organization because if there is no policy in regard to WRs then they leave themselves vulnerable to legal action that can occur if there is a messy breakup. It is suggested that workplaces should implement education relating to what behaviours are acceptable within their organization and whether or not it is legally sexual harassment, and ultimately just to be conscious of their own behaviours as employees (Chroy \& Hoke, 2019, p. 593).

It has also been suggested by Adler et al. (2014) that in order to avoid consequences related potential extramarital affairs management should attempt to involve spouses and significant others in events put on by the organization such as company parties or celebrations of retirement (p. 345). The existence of affairs in the workplace can cause chaos amongst employees and all precautions should be in place to keep them out of the workplace (p. 337).

\section{Conclusion}

The presence of intimate workplace relationships in organizations is not something that will be able to be completely controlled or eliminated. The workplace has become a common place to meet people in a romantic sense due to frequency of contact and potentially spending long hours with an individual in the pursuit of a common goal. Although inevitable, there are many complications that happen as a result of their existence. Depending on whether the relationship is hierarchal or lateral will affect how other employees of the organization perceive the relationship in the workplace. In the majority of cases relationships between subordinates and superiors are negatively perceived by co-workers due to the assumed favoritism and disproportionate opportunity the subordinate is expected to receive. The presence of WRs has also been discovered to decrease productivity and engagement within the workplace if the majority of co-workers have negative opinions in regard to their existence. Attitudes towards these relationships are influenced by factors such as motive, gender, age, workplace culture, and the manner in which employees found out their co-workers were in a relationship that was more than platonic. The creation of policy by management is essential in monitoring and regulating the presence of these relationships in the workplace as they are complex and are bound to create some complications between employees. An organization could be left vulnerable to legal complications if a couple within the 
workplace was to break up in manner that was not peaceful in which accusations of sexual harassment are not uncommon. Although there is a large amount of research that exists on this topic there is still much more contemporary information that is left to be discovered. For instance, there is very little substantial research regarding the existence of homosexual relationships in the workplace and how they are perceived. The research regarding workplace policy is quite theoretical and has the opportunity to be expanded upon. The presence of workplace relationships within any organization has the potential to affect everybody in the work force and warrants more research. 


\section{References}

Alder G. S., \& Quist D. M. (2014). Rethinking love at the office: Antecedents and consequences of coworker evaluations of workplace romances. Human Resource Management, 53, 3, 329-351. DOI: 10.1002/hrm.21572

Balaban R. V. (2019). Romance at work and the negative impact it has on the workplace environment. Journal of Public Administration, Finance \& Law, 15, 127-134.

Chroy R. M., \& Hoke H. G. G. (2019). Young love at work: Perceived effects of workplace romance among millennial generation organizational members. Journal of Psychology, 153, 6, 575-598. DOI: 10.1080/00223980.2019.1581722

Cowan R. L., \& Horan S. M. (2014). Love at the office? Understanding workplace romance disclosures and reactions for the coworker perspective. Western Journal of Communication, 78, 2, 238-253. DOI: 10.1080/10570314.2013.866688

Malachowski C.C., Chroy R. M., \& Claus C. J. (2012). Mixing pleasure with work: Employee perceptions of and responses to workplace romance. Western Journal of Communication, 76, 4, 358-379. DOI: 10.1080/10570314.2012.656215

Pierce C. A., Byrne D., \& Aguinis H. (1996). Attraction in organizations: A model of workplace romance. Journal of Organizational Behavior, 17, 1, 5-32.

Pierce C. A., \& Aguinis H. (1997). Bridging the gap between romantic relationships and sexual harassment in organizations. Journal of Organizational Behavior, 18, 3, 197-200.

Salvaggio A. M., Hopper J., \& Packell K. M. (2010). Coworker reactions to observing sexual behavior at work. Journal of Managerial Psychology, 26, 7, 604-622.

DOI:10.1108/02683941111164508

Shuck B., Owen J., Manthos M., Quirk K., \& Rhoades G. (2016). Co-workers with benefits. Journal of Management Development, 35, 3, 382-393. DOI: 10.1108/JMD-02-2015-0014

Wilson F. (2015). Romantic relationships at work: Why love can hurt. International Journal of Management Reviews, 17, 1, 1-19. DOI: 10.1111/ijmr.12034 